\title{
Detección de incendios forestales utilizando imágenes NOAA/16-LAC en la Región de La Araucanía, Chile
}

\author{
Forest fire detection using NOAA/16-LAC satellite images \\ in the Araucanía Region, Chile
}

\author{
Carlos Muñoz ${ }^{a *}$, Patricio Acevedo ${ }^{b}$, Sonia Salvo ${ }^{c}$, Gonzalo Fagalde ${ }^{a}$, Francisco Vargas ${ }^{d}$

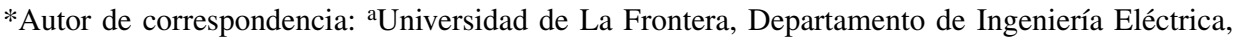 \\ Casilla 54-D, Temuco, Chile, fono: 56-45325531, fax: 56-45325550, comunoz@ufro.cl \\ ${ }^{\mathrm{b}}$ Universidad de La Frontera, Departamento de Ciencias Físicas, Temuco, Chile \\ c Universidad de La Frontera, Departamento de Matemática y Estadística, Temuco, Chile \\ d Corporación Nacional Forestal CONAF, IX Región, Chile
}

\begin{abstract}
SUMMARY
In this work, a perceptron neural network model for automatic forest fire detection based on satellite images is proposed. The model is aimed to be used at the region of La Araucanía in Chile. For this study, a set of 33 NOAA/16-AVHRR images corresponding to the 2004 forest fire season was used. The proposed classification method is based on a single layer perceptron neural network. The classification method uses the values of the five corresponding bands of each pixel of the satellite image as inputs plus the computed gradient of the infrared number 3 band of the satellite image. Both for the training stage and for the validation stage as well, statistical data were provided by the Chilean National Forest Corporation (CONAF). For the validation stage, forest fires as small as fifteen hectares in size were detected with $100 \%$ of accuracy, and a $50 \%$ of accuracy were obtained for forest fires as small as ten hectares.
\end{abstract}

Key words: forest fire, fire detection, satellite images, neural networks.

\section{RESUMEN}

El presente trabajo propone un modelo de red neuronal perceptron que utilizó datos de imágenes satelitales para detección automática de fuegos forestales. El modelo fue concebido para operar en la Región de La Araucanía, Chile. Para este estudio se utilizó un conjunto de 33 imágenes NOAA/16-AVHRR, correspondientes a las temporadas de incendios del año 2004. El método propuesto utilizó redes neuronales de tipo perceptron monocapa para el análisis de la imagen píxel a píxel. Las entradas a la red correspondieron a los valores del píxel en cada una de las cinco bandas del sensor satelital, más información del gradiente de temperatura en la banda infrarroja número 3. Tanto para el entrenamiento como para la validación se utilizó como salida información estadística de CONAF. En la etapa de validación se obtuvieron detecciones de fuegos de 15 ha con un $100 \%$ de efectividad, mientras con fuegos de 10 ha se obtuvo un $50 \%$ de efectividad, sin la presencia de nubes para ambas situaciones.

Palabras clave: detección de fuegos, incendios forestales, imágenes satelitales, redes neuronales.

\section{INTRODUCCIÓN}

La protección contra los incendios forestales es una actividad que en Chile ha sido realizada por más de 35 años, a través de distintas organizaciones estatales, siendo actualmente canalizada por intermedio de la Corporación Nacional Forestal (CONAF) dependiente del Ministerio de Agricultura. En el sector privado, las empresas forestales se sumaron a esta iniciativa, estableciendo sus propios sistemas de protección para la defensa de su patrimonio forestal, en estrecha colaboración con CONAF y su Departamento de Manejo del Fuego (DMF).

En Chile, el fuego normalmente es detectado usando monitoreo visual, desde torres de vigilancia localizadas estratégicamente en puntos altos con personal calificado que examina permanentemente el territorio a ojo desnudo o mediante largavistas. Además, se cuenta con vigilancia móvil, compuesta por motoristas y guardabosques que patrullan áreas reducidas y específicas, normalmente asociadas a zonas de alto riesgo y peligro de incendios forestales. 
Sin embargo, en sectores aislados y de difícil acceso, como por ejemplo en zonas cordilleranas de la costa o andina de la Región de La Araucanía, no es posible desarrollar una detección eficiente y efectiva con torres de vigilancia debido a la abrupta topografía, y mucho menos la detección móvil por la carencia de caminos adecuados. En la figura 1 se muestra un diagrama de la Región de La Araucanía con la marcas de localización de las torres de detección. En esta figura se puede apreciar que, si bien existe una buena cantidad de torres, también existen grandes zonas con escasa o simplemente nula cobertura de detección de incendios forestales. En la Región de La Araucanía existen 42 torres de vigilancia de fuegos forestales, considerando una distancia óptima de detección de $15 \mathrm{~km}$, la cobertura alcanza a 23,3\% del territorio regional.

Es en estos casos donde la información entregada por las imágenes satelitales es de gran utilidad para la teledetección de incendios forestales. Esto sigue siendo válido incluso si se considera que es un monitoreo que depende de la frecuencia de paso del satélite y de las características del sensor montado en él, tales como: la resolución espacial (tamaño del píxel), resolución espectral (número de bandas) y el ancho de dichas bandas.

En el ámbito internacional se ha realizado un gran avance debido a la preocupación por los incendios forestales (Martin et al. 1994) y sus efectos (Viedma y Chuvieco 1993, Chuvieco y Martin 2002, Heredia et al. 2003), lo cual se ve reflejado en los distintos mecanismos de detección, entre los cuales se destacan los que utilizan imágenes de satélite. Usar una imagen para localizar un fuego requiere de complejas técnicas de búsqueda, las cuales en su mayoría se basan en umbrales de temperatura (Zhukov y Lorenz 2006). Recientemente se ha propuesto con gran éxito el uso de redes neuronales para detectar automáticamente
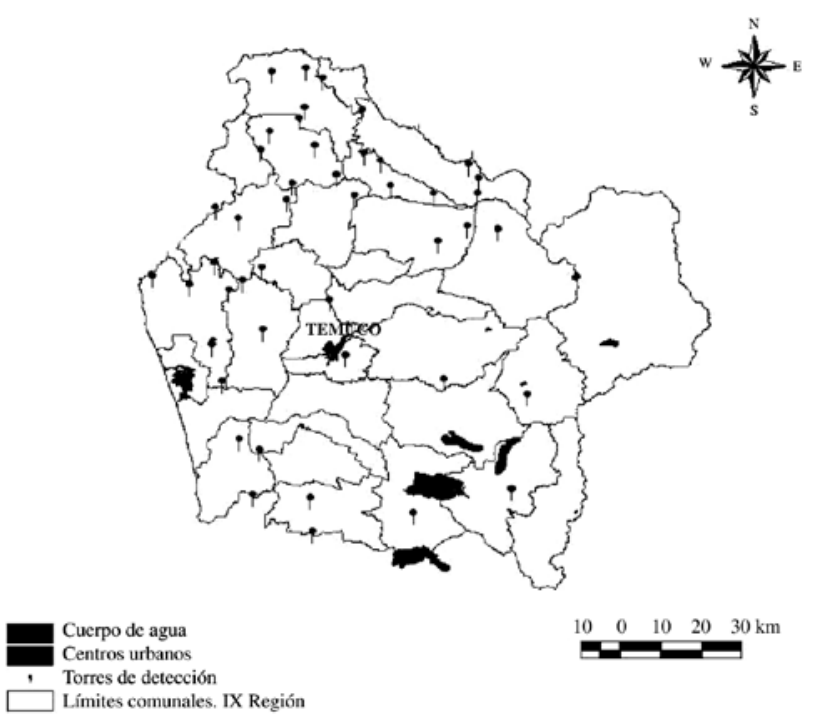

Figura 1. Mapa de ubicación geográfica de torres de obser vación.

Watching towers geographical location map. fuegos desde imágenes de satélite (Zhanqing et al. 2001, Gimeno y San Miguel 2004, Lee et al. 2004, Ono et al. 2005, Richards y Jia 2006).

En España se han incluido redes neuronales no sólo en la detección de incendios forestales, sino que además en sistemas de predicción de incendios utilizando categorías simbólicas de riesgo (Alonso et al. 2003). También han trabajado en esquemas de predicción-detección para disminuir errores en la detección utilizando sensores infrarrojos pasivos y sistemas no lineales (Bernabeu et al. 2004).

En Portugal, utilizando LIDAR (señales de radar en satélites de baja altura) en conjunto con redes neuronales de tipo perceptron monocapa, se han obtenido resultados exitosos al eliminar algunos inconvenientes que presenta la teledetección pasiva como lo son las nubes (Fernandes et al. 2004). También en China se han realizado trabajos enfocados al monitoreo de la dinámica forestal a través de teledetección (Liu et al. 2005). En Chile, los estudios realizados hasta el momento no presentan reconocimiento ni clasificación automática de incendios forestales.

Cabe destacar que los métodos de detección usados con éxito son aplicados a imágenes satelitales que contienen bandas infrarrojas (Zhukov y Lorenz 2006). Entre los satélites más usados que entregan este tipo de imágenes se encuentran el NOAA/16-AVHRR y el TERRA-MODIS (Mitchell 1996, Kant et al. 2000, Zhanqing y Khananian 2001, Asakuma et al. 2002, Chuvieco y Martin 2002, Chuvieco et al. 2004, Jin y Sader 2005, Sabins 1997, Ru et al. 2000, Siegert y Hoffmann 2000, Sifakis et al. 2004, Sukhinin et al. 2004).

El sensor NOAA/16-AVHRR, instalado en el satélite NOAA, es el más utilizado para la teledetección de incendios forestales debido a sus tres bandas térmicas. Este sensor tiene una frecuencia de paso de $12 \mathrm{~h}$, pero sólo se tiene acceso a una pasada diaria, su resolución espacial es de $1,1 \mathrm{~km}^{2}$, es decir, aproximadamente 100 hectáreas, y registra información en cinco bandas. Dossier (1981) demuestra que aun cuando una fuente de alta temperatura afecte sólo a una porción de un píxel, es posible de ser detectada, ya que provoca un notable incremento de la radiación emitida en las longitudes de onda del infrarrojo. Un ejemplo de esto lo presenta Wright et al. (2002), indicando que en el caso del infrarrojo cercano (banda 3 del sensor AVHRR-NOAA) una fuente a una temperatura de $580{ }^{\circ} \mathrm{C}$, ocupando sólo el $0,05 \%$ de un píxel $\left(500 \mathrm{~m}^{2}\right)$, genera un aumento por sobre el $200 \%$ de la energía infrarroja emitida. Lo anterior ha sido utilizado como base en el monitoreo volcánico (Pergola et al. 2004).

Por lo expuesto anteriormente, el objetivo de esta investigación es proponer un modelo de red neuronal perceptron que utiliza datos de imágenes satelitales para detección automática de fuegos forestales. Se hipotetiza que, dado que el sensor NOAA/16-AVHRR tiene una resolución espacial de $1,1 \mathrm{~km}^{2}$, el modelo es capaz de detectar fuegos forestales en una superficie menor a la abarcada por un píxel. 


\section{MÉTODOS}

El área de estudio correspondió a la Región de La Araucanía de Chile; superficie aproximada de $31.858 \mathrm{~km}^{2}$, representando el 4,2\% del territorio nacional continental. Se ubica entre los $37^{\circ} 35^{\prime}$ y $39^{\circ} 37^{\prime}$ latitud Sur y desde los $70^{\circ} 50^{\prime}$ longitud Oeste, como coordenadas geográficas, y entre los 629.500 y los 864.000 UTM latitud Sur; 5.607.500 y 5.839.000 UTM longitud Oeste, correspondientes al Datum provisional sudamericano Sur Chile de 1956, huso 18 Sur extendido.

Las imágenes NOAA/16-AVHRR se obtuvieron en forma gratuita ingresando a la página web http://www. saa.noaa.gov, donde previo registro se realizó el pedido de imágenes, seleccionando la fecha, lugar geográfico y satélite. Las características espectrales para cada banda del sensor NOAA/16-AVHRR se presentan en el cuadro 1.

Para el presente trabajo se utilizaron 33 imágenes del satélite NOAA/16-AVHRR entre el 1 de noviembre de 2003 y el 31 de marzo de 2004. Como ejemplo se presenta en la figura 2 la imagen del 13 de enero de 2004 en su banda 3. Además, se utilizó la base de datos de ocurrencia y daño de incendios forestales del DMF de CONAF para la temporada 2004, y que se usó en este trabajo (cuadro 2). Las 33 imágenes satelitales fueron calibradas a reflectancia (banda 1 y banda 2) y a temperatura de brillo (banda 3 , banda 4 y banda 5). Además, las imágenes se referenciaron geográficamente, a fin de hacerlas compatibles con los SIG existentes, entre ellos el empleado por CONAF. En el cuadro 3 se indican las fechas de las imágenes.

El software empleado para el procesamiento de las imágenes de satélite es ENVI 4.0, y para la implementación de las redes neuronales es MATLAB 6.5. En el procesamiento inicial de las imágenes satelitales no se consideró la aplicación de correcciones atmosféricas, ya que todas las imágenes corresponden a un mismo período de verano, con lo cual es posible asumir condiciones atmosféricas similares.

Para la calibración radiométrica se utilizaron los parámetros incluidos en el archivo entregado por el NOAA/16AVHRR, que corresponden a un header y a las 5 bandas del sensor. Para la corrección espacial (georreferenciación) se utilizó el método de transformación "triangulación", realizando el remuestreo de la imagen con el método del

Cuadro 1. Características espectrales del sensor NOAA/ 16-AVHRR.

Spectral characteristics of the AVHRR-NOAA/16 sensor.

\begin{tabular}{cc}
\hline Bandas & Longitud de onda $(\mathrm{nm})$ \\
\hline 1 & $580-680$ \\
2 & $725-1.100$ \\
3 & $3.550-3.930$ \\
4 & $10.300-11.300$ \\
5 & $11.500-12.500$ \\
\hline
\end{tabular}

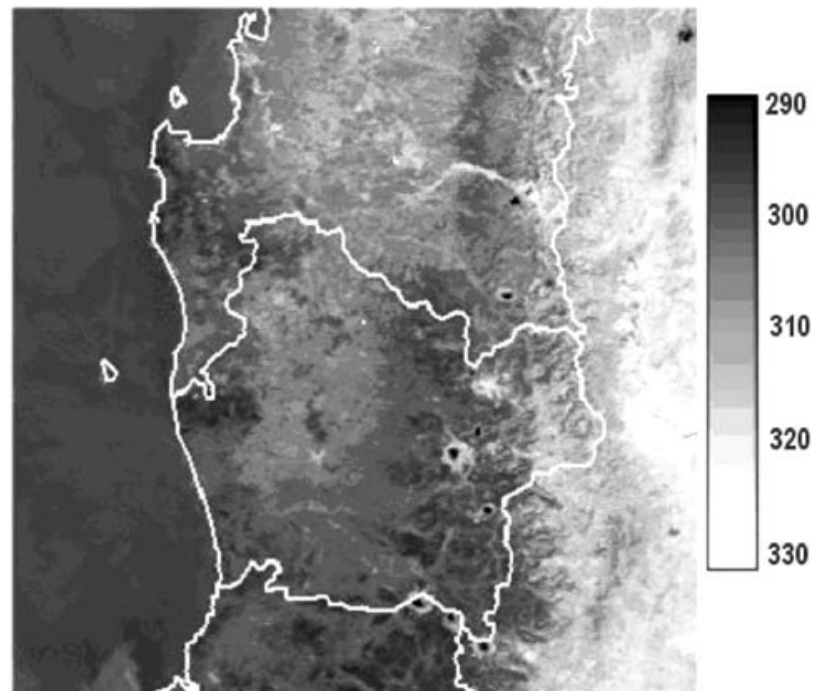

Figura 2. Imagen NOAA/16-AVHRR del 13 de enero de 2004 calibrada a temperatura de brillo y georreferenciada. La paleta de grises representa las temperaturas de brillo en ${ }^{\circ} \mathrm{K}$.

NOAA/16-AVHRR image on January 13 2004, calibrated at bright temperature and geo-referenced. The grey pallet represents the bright temperature in ${ }^{\circ} \mathrm{K}$.

Cuadro 2. Base de datos de ocurrencia y daño de incendios forestales de CONAF para la temporada noviembre 2004 a marzo 2005. Fuente: Estadística CONAF IX Región.

CONAF data base with the occurrence and damage of forest fires for the season November 2004 to March 2005. Source: Statistic CONAF IX Region.

\begin{tabular}{|c|c|c|}
\hline Lugar & $\begin{array}{c}\text { Superficie } \\
\text { quemada (ha) }\end{array}$ & $\begin{array}{c}\mathrm{N}^{\mathrm{o}} \mathrm{de} \\
\text { Incendios }\end{array}$ \\
\hline Región de La Araucanía & $6.266,16$ & 1.275 \\
\hline Provincia Malleco & $4.874,44$ & 952 \\
\hline Comuna Angol & 393,78 & 158 \\
\hline Comuna Collipulli & $1.468,54$ & 274 \\
\hline Comuna Curacautín & 0,02 & 2 \\
\hline Comuna Ercilla & 280,05 & 133 \\
\hline Comuna Lonquimay & 7,50 & 1 \\
\hline Comuna Los Sauces & 388,60 & 31 \\
\hline Comuna Lumaco & 930,13 & 119 \\
\hline Comuna Purén & 25,62 & 37 \\
\hline Comuna Renaico & 35,58 & 17 \\
\hline Comuna Traiguén & 422,84 & 48 \\
\hline Comuna Victoria & 921,78 & 132 \\
\hline Provincia Cautín & $1.391,72$ & 323 \\
\hline Comuna Carahue & 30,85 & 20 \\
\hline Comuna Cholchol & 47,44 & 12 \\
\hline Comuna Cunco & 0,01 & 1 \\
\hline Comuna Curarrehue & 0,80 & 2 \\
\hline Comuna Freire & 11,46 & 5 \\
\hline Comuna Galvarino & 430,76 & 52 \\
\hline Comuna Gorbea & 3,30 & 6 \\
\hline Comuna Lautaro & 250,36 & 21 \\
\hline Comuna Loncoche & 9,74 & 9 \\
\hline Comuna Melipeuco & 0,30 & 1 \\
\hline Comuna Nva. Imperial & 10,19 & 9 \\
\hline Comuna P. Las Casas & 23,98 & 18 \\
\hline Comuna Perquenco & 352,83 & 9 \\
\hline Comuna Pitrufquén & 10,10 & 7 \\
\hline Comuna Pucón & 15,31 & 2 \\
\hline Comuna Temuco & 117,94 & 132 \\
\hline Comuna Toltén & 0,70 & 1 \\
\hline Comuna Vilcún & 67,24 & 6 \\
\hline Comuna Villarrica & 8,41 & 10 \\
\hline
\end{tabular}


Cuadro 3. Fechas de adquisición de las imágenes NOAA/16AVHRR utilizadas en el presente trabajo.

Dates of the acquisition for the NOAA/16-AVHRR images used in this work.

\begin{tabular}{ccccc}
\hline \multicolumn{2}{c}{2003} & \multicolumn{3}{c}{2004} \\
\hline Noviembre & Diciembre & Enero & Febrero & Marzo \\
\hline 01 & 08 & 03 & 01 & 03 \\
02 & 15 & 11 & 09 & 12 \\
03 & 16 & 12 & 11 & 15 \\
04 & 17 & 13 & 15 & 21 \\
05 & 19 & 20 & 16 & \\
09 & 23 & 30 & 18 & \\
19 & 24 & & 25 & \\
28 & 28 & & & \\
\hline
\end{tabular}

Vecino más Cercano, a fin de conservar el valor radiométrico original. Ambos procedimientos vienen incluidos en el software ENVI 4.0. El sistema de coordenadas utilizado es el UTM WGS84 huso 18 Sur.

Cada banda posee un número definido de píxeles, los cuales se designaron de la siguiente forma: $P_{i, j}^{k}$, donde $k$ representa la banda y el par $i, j$ representan la columna y fila, respectivamente en la imagen. Esto es,

$$
P_{i, j}^{k} \in \Re, i \in\{1, \ldots, 200\}, j \in\{1, \ldots, 250\}, k \in\{1, \ldots, 5\}
$$

Para las primeras dos bandas los valores de píxel $P_{i, j}^{1}$ y $P_{i, j}^{2}$ representan reflectancias (escaladas entre 0 y 100 ). Para las restantes bandas, los valores de píxel $P_{i, j}^{3}, P_{i, j}^{4}$ y $P_{i, j}^{5}$ representan temperaturas de brillo en el rango de 180 a $335^{\circ} \mathrm{K}$.

En este trabajo se propuso un modelo de detección que utiliza los datos de las 5 bandas del sensor NOAA/16AVHRR para cada uno de los píxeles de las imágenes. Cada píxel representa el estado de un sector de 100 hectáreas de terreno. Los datos de cada una de las bandas, en sus diferentes píxeles, se utilizaron como entradas de una sola neurona del tipo perceptron, y su salida correspondió a la presencia o ausencia de fuego en el sector analizado.

Para aumentar la sensibilidad del sistema se procedió a incorporar una nueva variable $N_{i, j}$, que contiene la información del gradiente de temperatura para el canal 3 del sensor (figura 3). El valor de $N_{i, j}$ se consiguió usando una ventana de medias móviles que promedia los 8 píxeles más cercanos a $P_{i, j}^{3}$, con lo cual, los $N_{i, j}$ se obtuvieron según la ecuación [2], con $i=2,3, \ldots, 199 ; j=2,3, \ldots, 249$.

$N_{i, j}=\frac{P_{i-1, j-1}^{3}+P_{i, j-1}^{3}+P_{i+1, j-1}^{3}+P_{i-1, j}^{3}+P_{i+1, j}^{3}+P_{i-1, j+1}^{3}+P_{i, j+1}^{3}+P_{i+1, j+1}^{3}}{8}$

De este modo, el modelo neuronal quedó como el representado en la figura 4 o, equivalentemente, el descrito en la ecuación [3], donde $Y_{i, j}^{m}$ son las salidas binarias que representan la ausencia o presencia de fuego

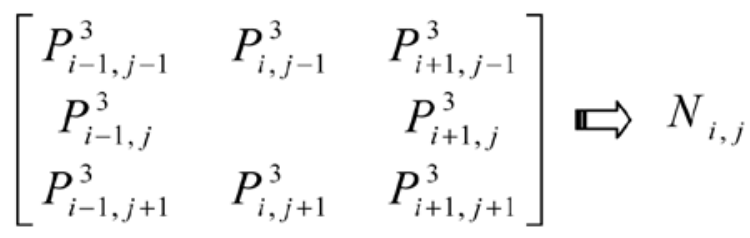

Figura 3. Ventana de medias móviles. Moving average window.

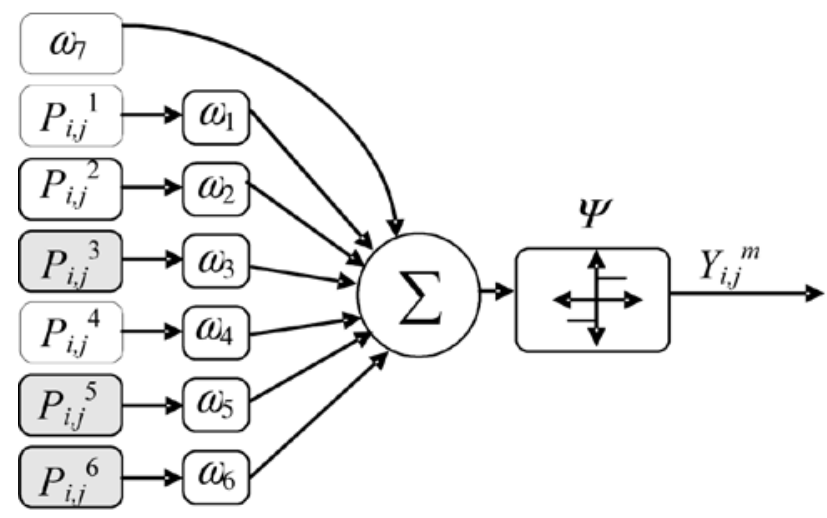

Figura 4. Red neuronal.

Neural network.

y $\operatorname{los}\left(\omega_{i}, i=1, \ldots, 7\right)$ son pesos que se obtienen del entrenamiento de la red y $\Psi$ es una función de activación de tipo signo.

Nótese que, una vez entrenado el modelo, la nueva imagen depende sólo de los niveles de gris de cada banda de la imagen satelital (ver ecuación [3]).

$$
Y_{i, j}^{m}=\Psi\left[\sum_{i=1}^{5} \omega_{i} P_{i, j}^{i}+\omega_{6} N_{i, j}+\omega_{7}\right]
$$

El entrenamiento de la red busca que los parámetros del modelo $\left(\omega_{i}, i=1 \ldots, 7\right)$ de la neurona entrenada (ecuación [3]), sean tales que la salida de la red neuronal $Y_{i, j}^{m}$ sea idéntica a la imagen de detección real $Y_{i, j}$, es decir, a los fuegos detectados en el área, fecha y hora a la cual corresponde la imagen satelital.

Para componer la imagen de detección real, $Y_{i, j}$, se utilizó información recopilada en terreno por el DMF de CONAF. Esta información consideró la ubicación en coordenadas UMT $(x, y)$, la fecha de inicio del incendio, hora de inicio, fecha de término, hora de término y tamaño del daño causado. Esta información fue ordenada a fin de construir una matriz exportable a MATLAB.

Visto que la resolución espacial del sensor NOAA/16AVHRR es de $1,1 \mathrm{~km}^{2}$, se consideró el píxel de 100 hectáreas correspondiente a la ubicación $i, j$ con condición de fuego, cuando se tiene al menos un incendio informado que involucra un área mayor a 0,5 hectáreas. Bajo condición de fuego, se asigna un valor 1 a $Y_{i, j}$ y 0 en otro caso. 
Para el entrenamiento de la red neuronal se importó cada una de las imágenes al software MATLAB. Mediante análisis visual y utilizando información extraída de las estadísticas de CONAF para la fecha correspondiente, se identificaron en la imagen satelital los píxeles informados como fuego y que presentaron alta temperatura en el canal 3 del sensor NOAA/16-AVHRR. Se seleccionaron algunas filas completas de la imagen que contenían píxeles marcados con condición de fuego. Se utilizaron filas y no columnas con el objetivo de incorporar la mayor variabilidad EsteOeste de la región geográfica. En el entrenamiento de la red neuronal los mejores resultados se obtuvieron usando cuatro filas de la imagen satelital en las cuales existían incendios informados por CONAF que correspondían al menos a dos píxeles por fila, ya que un número mayor de filas no aporta mayor información. Conviene decir que cuatro filas de una imagen constituyen aproximadamente 2.000 datos de entrada para el entrenamiento de la red neuronal.
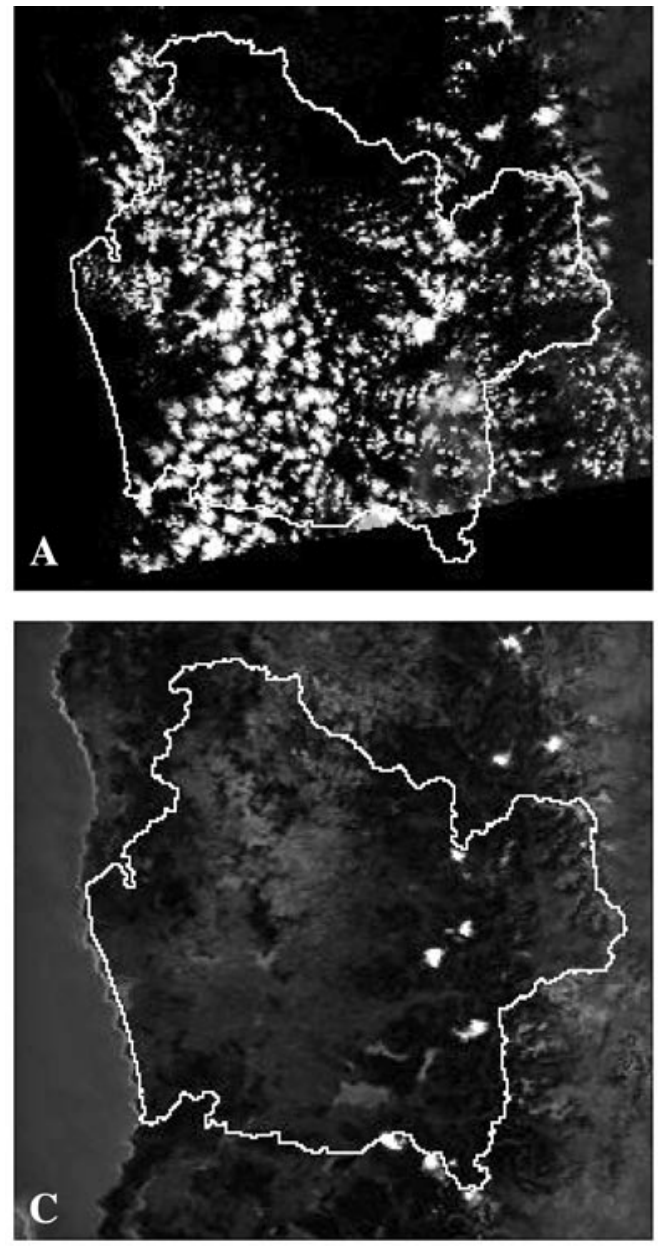

\section{RESULTADOS}

Los resultados obtenidos a partir de la detección de incendios con redes neuronales se validaron con las estadísticas de CONAF. Para esto se seleccionaron tres imágenes, considerando sólo aquellas con la menor cobertura de nubes y en las que la zona estudiada correspondiera a ángulos satelitales de observación menores a $25^{\circ}$ : 28-dic-2003, 13-ene-2004 y 03-mar-2004 (figura 5). En general, para el período estudiado las condiciones de cobertura de nubes es la presentada en la figura $5 \mathrm{a}$.

Los resultados de la detección de incendios y aquellos informados por CONAF para los días 13 de enero de 2004 y 3 de marzo de 2004, se presentan en las figuras 6 y 7. En cada una de ellas se indican con rombos (blancos o negros) los incendios detectados e informados. En el cuadro 4 , se entregan los porcentajes de detección correcta de incendios forestales.
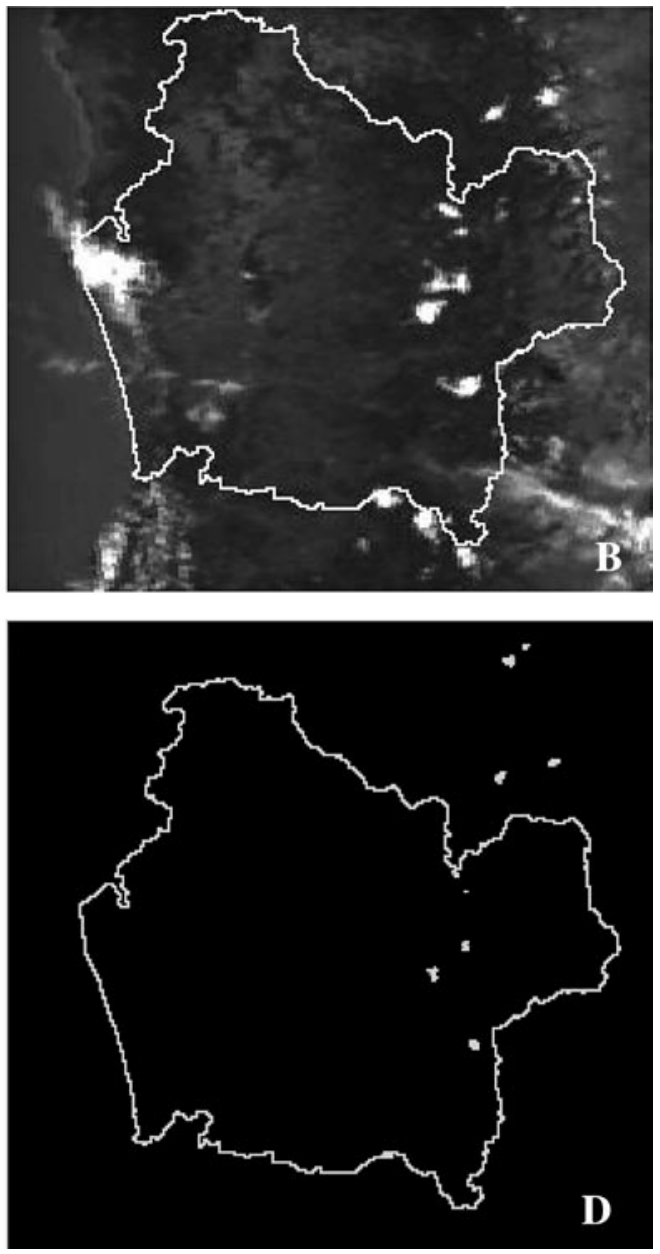

Figura 5. Detección de nubes para las imágenes del: (A) 15-12-2003; (B) 28-12-2003; (C) 13-01-2004; (D) 03-03-2004. Las áreas blancas representan nubes o nieve, mientras que la línea continua blanca corresponde al borde regional.

Clouds detection on images at: (A) 15-12-2003; (B) 28-12-2003; (C) 13-01-2004; (D) 03-03-2004. The white area represents clouds or snow, while the continuous line corresponds to the border between geographical regions. 

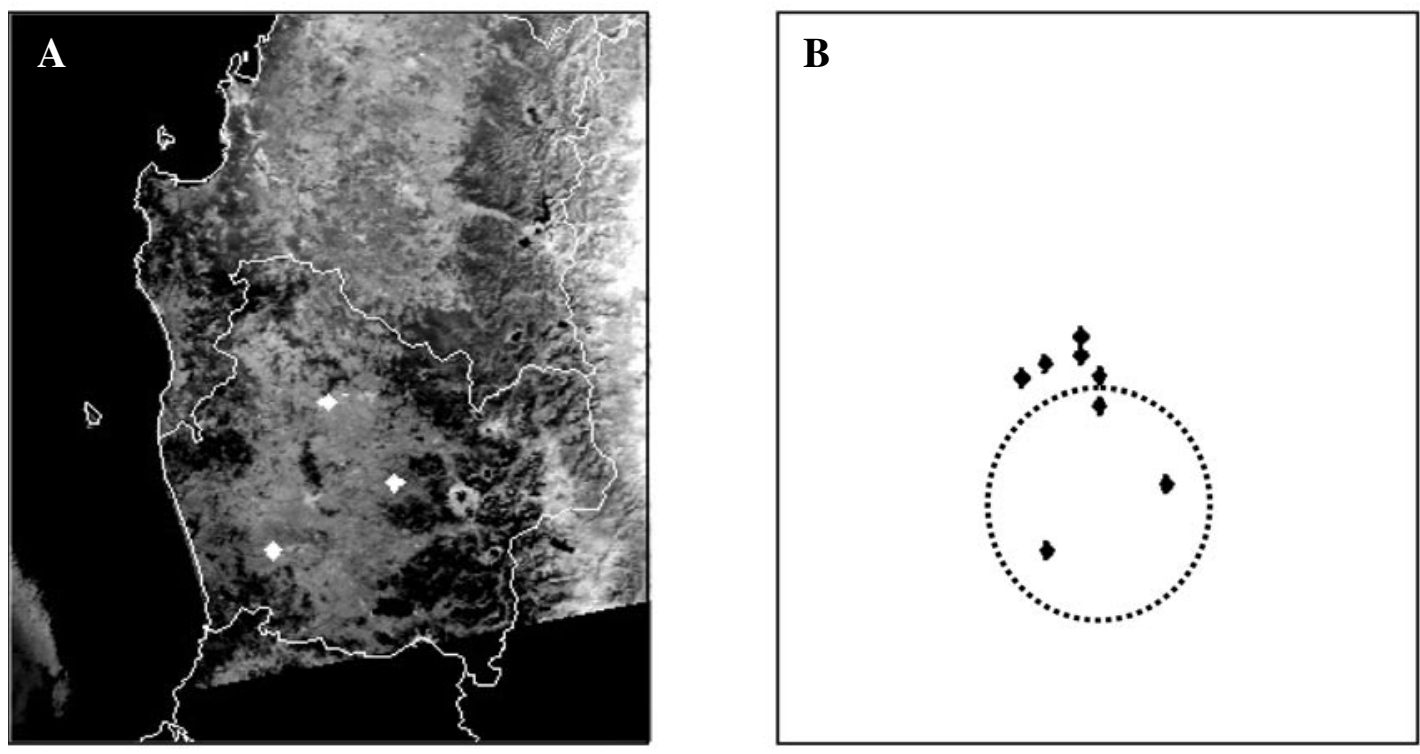

Figura 6. (A) En la figura de la izquierda se muestran los resultados de la detección de fuego para focos mayores de 5 ha, en el día 3 de marzo de 2004, representados con puntos blancos sobre la imagen NOAA/16AVHRR. (B) En la figura de la derecha, con puntos negros se muestran los incendios reportados por CONAF. La línea punteada encierra el total de incendios detectados.

(A) Result for fire forest detection for areas bigger than 5 ha, on March 3 of 2004 is shown by white marks on the image AVHRR-NOAA; (B) Fire forests reported by CONAF are shown by black marks. The dotted line encircles the fire forests well detected.
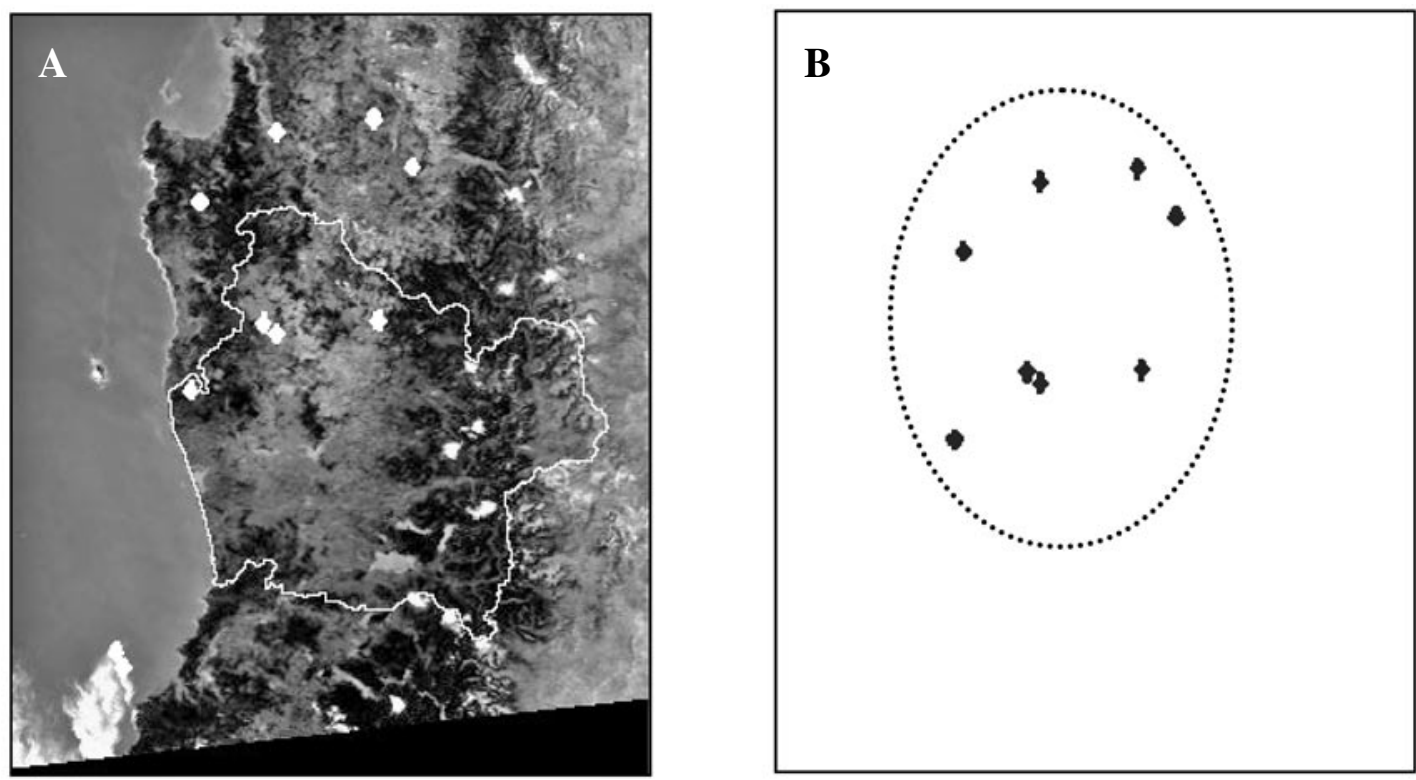

Figura 7. (A) En la figura de la izquierda se muestran los resultados de la detección de fuego para focos mayores de 10 ha, en el día 13 de enero de 2004, representados con puntos blancos sobre la imagen NOAA/16AVHRR. (B) En la figura de la derecha, con puntos negros se muestran los incendios reportados por CONAF. La línea punteada encierra el total de incendios detectados.

(A) Result for fire forest detection for areas bigger than 5 ha, on January 13 of 2004 is shown by white marks on the image AVHRR-NOAA; (B) Fire forests reported by CONAF are shown by black marks. The dotted line encircles the fire forests well detected. 
Cuadro 4. Porcentaje de detección correcta de incendios forestales usando imágenes NOAA/16-AVHRR.

Percentage of correct forest fire detection using NOAA/16-AVHRR images.

\begin{tabular}{lrrrrrrr}
\hline \multirow{2}{*}{ Fechas } & \multicolumn{7}{c}{ Porcentaje de incendios detectados } \\
\cline { 2 - 8 } & $<0,5$ ha & $<1$ ha & $<5$ ha & $<10$ ha & $<15$ ha & $<20$ ha & $>20$ ha \\
\hline $28 / 12 / 2003$ & 33 & 100 & 100 & 100 & 100 & 100 & 100 \\
$13 / 01 / 2004$ & 10 & 21 & 33 & 100 & 100 & 100 & 100 \\
$3 / 03 / 2004$ & 13 & 25 & 38 & 50 & 100 & 100 & 100 \\
\hline
\end{tabular}

\section{DISCUSIÓN}

Si bien es posible identificar a ojo desnudo un fuego forestal de 100 ha en una imagen satelital (obtenida desde el sensor NOAA/16 AVHRR), esto es una tarea compleja y que puede llevar fácilmente a errores de detección, en particular en el caso que se esté haciendo un análisis visual de las diferentes bandas de una imagen satelital. Si en un píxel específico de la imagen se detecta una subida de sólo un par de grados en la banda 3 y simultáneamente, una leve disminución de la reflectancia en la banda 1 puede significar que hay un incendio de 100 ha, aunque dichos cambios no alteren perceptiblemente la representación del color de los píxeles analizados.

Esto ocurre debido a que a pesar de las grandes extensiones que puede abarcar un incendio forestal, éste no sólo está compuesto por fuego que presenta una alta temperatura y que es fácil de detectar con un simple análisis visual, sino que también involucra superficies quemadas con una temperatura menor, por lo cual la detección se vuelve mucho más complicada considerando la resolución espacial del sensor NOAA/16-AVHRR. En este caso, una proporción importante de un píxel cubre superficies quemadas (de baja reflectancia y temperatura no muy alta) y sólo una parte reducida corresponde a fuego (con valores altos de reflectancia y temperatura), con lo cual el promedio del píxel presenta sólo un pequeño incremento en la temperatura y una leve tendencia a ser más reflectivo.

Otro aspecto a considerar es la evolución del incendio forestal. A partir de los antecedentes proporcionados por CONAF se tiene la extensión del área quemada para cada incendio. Sin embargo, en su momento de inicio (aun cuando haya comenzado antes del paso del satélite) es de tamaño reducido y de difícil detección. Por lo tanto, el umbral de detección de fuegos depende fuertemente de la temperatura de combustión del incendio, tanto como del contraste térmico que presenten las áreas cubiertas con fuego respecto del área total de un píxel (Martin $e t$ al. 1994). Asimismo, es importante hacer notar que la metodología empleada requiere la no saturación de las bandas utilizadas, especialmente la 3 y 4 (Setzer y Pereira 1991 y Pereira et al. 1991), lo que se tuvo presente durante el procesamiento de las imágenes utilizadas en el presente trabajo.

Los resultados obtenidos en el presente trabajo, con un $100 \%$ de detección para incendios mayores a 15 ha, superan a los informados aplicando métodos tradicionales de detección de incendios (Kant et al. 2000, Setzer y Pereira 1991, Sukhinin et al. 2004) basados en un análisis multibanda con aplicaciones de valores umbrales establecidos experimentalmente y modificados de acuerdo a las características de cada zona en estudio. Por ejemplo, utilizando imágenes del sensor AVHRR/NOAA, Sukhinin et al. (2004) informan que incendios con extensión menor a 1.000 ha no fueron detectados. Li et al. (1997) y Boles y Verbyla (2000), utilizando el mismo sensor, informan detección del $80 \%$ y $57 \%$ para el caso de incendios con extensiones mayores a 200 y 400 ha, respectivamente. Zhanking et al. (2001) utilizan redes neuronales similares a las utilizadas en este trabajo, pero sólo con los canales 1 a 5 como entradas, para reconocer plumas de humo en imágenes obtenidas con el sensor AVHHR y el TOMS, y establecen que las señales detectadas son muy diferentes, aunque se complementan para lograr una adecuada detección. La detección lograda por Zhanking se reduce a 1 píxel, lo que es superado ampliamente en el presente trabajo con la inclusión de una entrada adicional que representa el gradiente de temperatura en el canal 3, llegando a detecciones exitosas en un área cubierta por sólo el 15\% de un píxel. Otros resultados obtenidos con redes neuronales, pero para detectar humos con LIDAR, alcanzaron detecciones sobre el $90 \%$ en fuegos pequeños (Fernandes et al. 2004).

Además de la dificultad de detección generada por la resolución espacial del sensor NOAA/16-AVHRR, las nubes son el gran problema en la teledetección con sensores pasivos, debido que al cubrir la superficie terrestre, enmascara toda información proveniente de ella. Esto ha sido ampliamente informado en la literatura (Flannigan y Von der Haar 1986, Setzer y Pereira 1991). Por esta razón, en la selección de imágenes se escogieron las que presentaban el menor porcentaje de nubes, sin embargo, en la mayoría de las imágenes están presentes las nubes. $\mathrm{Al}$ aplicar el modelo propuesto para la detección de fuego, 

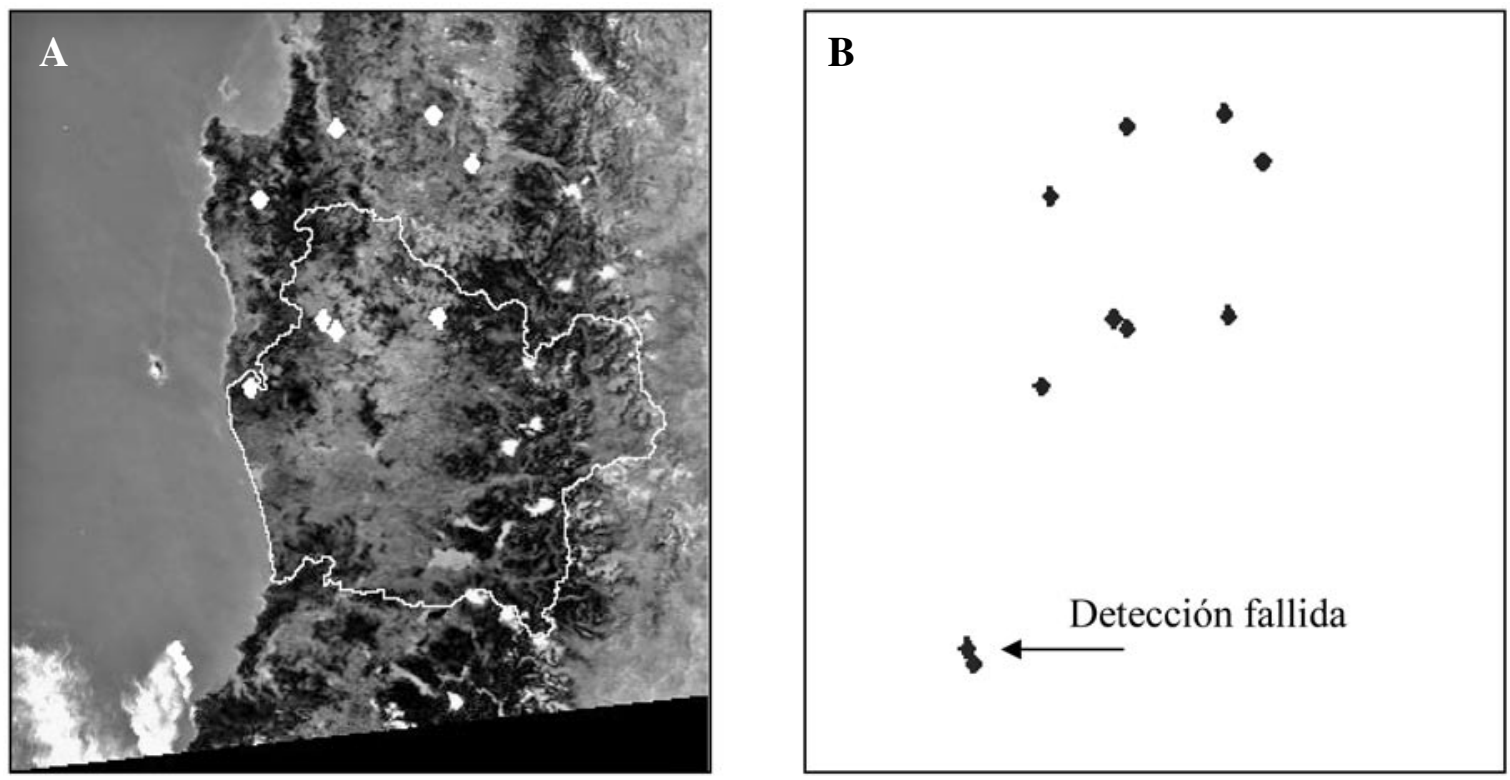

Figura 8. Resultados de la detección de fuego para el 13 de enero de 2004: (A) en la figura de la izquierda se presentan como rombos blancos sobre la imagen NOAA/16-AVHRR; (B) en la figura de la derecha como rombos negros a fin de resaltar sus posiciones. En el extremo inferior izquierdo de la imagen NOAA/16-AVHRR se aprecian nubes, las cuales son detectadas erróneamente (efecto borde) por el sistema como puntos de fuego.

Result for fire forest detection on January 13 of 2004, (A) on the left image white marks is used to enlighten the detected fire location on the image AVHRR-NOAA; (B) on the right image, black marks are used to show the locations of the fire forest reported by CONAF. The dotted line encircles the fire forests well detected. In the left-down corner of the AVHR-NOAA image some clouds are wrongly detected as fire forests (border effect).

las nubes generaron detecciones fallidas (figura 8), a pesar de que fue entrenado para reconocerlas, lo que no funcionó exitosamente debido, probablemente, a su alta variabilidad. Por lo anterior, para la validación del método propuesto se escogieron exclusivamente las mejores imágenes de cada mes, buscando la ausencia total de nubes a fin de evitar posibles errores en la detección.

En el caso de presencia de nubes, toda la información que evidencia un incendio desaparece debajo de la nube, con lo cual la detección mediante el uso de sensores pasivos como el NOAA/16-AVHRR no se aplica. Es más, las falsas detecciones originadas por la presencia de nubes son fácilmente distinguibles a ojo desnudo, con lo cual pueden ser descartadas sin problemas.

El modelo propuesto es una potente herramienta que permite apoyar la detección de focos de fuegos forestales a partir del análisis de imágenes entregadas por el sensor NOAA/16-AVHRR. Esto se traduce en disminuir el tiempo y mejorar la precisión de estas detecciones. La información resultante es especialmente útil si corresponde al $88 \%$ del territorio de la Región de La Araucanía que no está cubierto por el sistema de torres de vigilancia de fuegos forestales, ya que dicho territorio es cordillerano, con una densa cubierta de árboles y de difícil acceso, con lo que cualquier verificación requiere usualmente el uso de helicópteros o aviones.

\section{CONCLUSIONES}

El modelo propuesto, que se basó en una red neuronal perceptron de seis entradas, es una alternativa eficiente para la detección automática de fuegos o puntos de calor para superficies superiores a 15 ha, logrando un reconocimiento del $100 \%$ sin la presencia de nubes. En caso contrario puede generar falsas alarmas debido a la alta reflectancia que presentan las nubes en los canales 1 y 2 del sensor NOAA/16-AVHRR.

La selección de los datos para entrenamiento de las redes neuronales fue influyente en los resultados obtenidos, por lo cual es necesario efectuar un importante número de entrenamientos y comparar sus resultados. Esto conlleva necesariamente un gran tiempo de procesamiento, ligado tanto al número de entrenamientos propuestos, al tamaño de las imágenes a ser procesadas y a la capacidad de la máquina que se esté usando para dicho efecto.

El tiempo de un entrenamiento de la red neuronal fue de aproximadamente una hora, mientras que el tiempo de detección con una red entrenada no sobrepasó los cinco minutos. Los tiempos de entrenamiento de las redes neuronales están sujetos no sólo a la cantidad de datos necesarios para el entrenamiento, sino también a la calidad de éstos, entendiéndose por calidad a qué tan claras son las diferencias entre los datos a identificar y los datos restantes. 


\section{AGRADECIMIENTOS}

Este trabajo se realizó gracias al financiamiento entregado por la Universidad de La Frontera, casilla 54-D, al proyecto DIUFRO No 120614.

\section{REFERENCIAS}

Alonso A, O Fontenla, B Guijarros, E Hernández, M Andrade, E Jiménez, J Legido, T Carballas. 2003. An intelligent system for forest fire risk prediction and fire fighting management in Galicia. Expert Systems with Applications 25(4): 54-554.

Asakuma K, H Kuze, N Takeuchi, T Yahagi. 2002. Detection of biomass burning smoke in satellite images using texture analysis. Atmospheric Environment 36(9): 1531-1542.

Bernabeu P, L Vergara, I Bosh, J Igual. 2004. A prediction/detection scheme for automatic forest fire surveillance. Digital Signal Processing 14(5): 481-507.

Boles S, D Verbyla. 2000. Comparison of Three AVHRR-Based Fire Detection Algorithms for Interior Alaska. Remote Sensing of Environment 72: 1-16.

Chuvieco E, D Cocero, D Riaño, P Martin, J Martínez, J de la Riva, F Pérez. 2004. Combining NDVI and surface temperature for the estimation of live fuel moisture content in forest fire danger rating. Remote Sensing of Environment 92(3): 322-331.

Chuvieco E, MP Martin. 2002. Evaluación de imágenes NOAAAVHRR y TERRA-MODIS para cartografía regional de áreas quemadas. X Simposio Internacional de la Sociedad de Expertos Latinoamericanos en Percepción Remota (SELPER). $10 \mathrm{p}$.

Dossier J. 1981. A method for satellite identification of surface temperature fields of subpixel resolution. Remote Sensing of Environment 11: 221-229.

Fernandes A, A Utkin, A Lavrov, R Vilar. 2004. Development of neural network committee machines for automatic forest fire detection using lidar. Pattern Recognition 37(10): 2039-2047.

Flannigan M, T Von der Haar. 1986. Forest FIRE monitoring using NOAA satellite AVHRR. Canadian Journal of Forest Research 16: 975-982.

Gimeno M, J San Miguel. 2004. Evaluation of RADARSAT-1 data for identification of burnt areas in Southern Europe. Remote Sensing of Environment 92(3): 370-375.

Heredia A, S Martínez, E Quintero, W Piñeros, E Chuvieco. 2003. Comparación de distintas técnicas de análisis digital para la cartografía de áreas quemadas con imágenes LANDSAT ETM+. Geofocus 1: 216-234.

Jin S, S Sader. 2005. MODIS time-series imagery for forest disturbance detection and quantification of patch size effects. Remote Sensing of Environment 99(4): 462-470.

Kant Y, V Prasad, K Badarinath. 2000. Algorithm for detection of active fire zones using NOAA AVHRR data. Infrared Physics \& Technology 41(1): 29-34.

Lee E, R Yuen, S Lo, K Lam, G Yeoh. 2004. A novel artificial neural network fire model for prediction of thermal interface location in single compartment fire. Fire Safety Journal 39(1): 87.
Li Z, J Cihlar, L Moreau, F Huang, B Lee. (1997). Monitoring of fire activities in the boreal ecosystem. J. Geophys. Res. 102: 29611-29624.

Liu Q, X Li, Z Ma, N Takeuchi. 2005. Monitoring forest dynamics using satellite imagery: a case study in the natural reserve of Changbai Mountain in China. Forest Ecology and Management 210(1-3): 25-37.

Martin M, E Chuvieco, L Domínguez. 1994. Cartografía, evaluación y seguimiento de grandes incendios forestales a partir de imágenes NOAA-AVHRR. Revista de Teledetección. Revista de la Asociación Española de Teledetección 3: 1-6.

Mitchell R. 1996. Preflight calibration anomaly in the NOAA 14 AVHRR Channels 1 and 2. Remote Sensing of Environment 56(2): 141-147.

Ono T, H Ishii, K Kawamura, H Miura, E Momma, T Fujisawa, J Hozumi. 2005. Application of neural network to analyses of CCD colour TV-camera image for the detection of car fires in expressway tunnels. Fire Safety Journal 41: 279-284.

Pereira A, A Setzer, J Dos Santos. 1991. FIRE estimates in savannas of central Brazil with termal AVHRR/NOAA calibrated by TM/Landsat. Proc. 24th International Symposium on Remote Sensing of Environment. Río de Janeiro, Brazil, p. $825-836$.

Pergola N, F Marchese, V Tramutoli. 2004. Automated detection of thermal features of active volcanoes by means of infrared AVHRR records. Remote Sensing of Environment 93: 311-327.

Richards J, X Jia. 2006. Remote Sensing Digital Image Analysis: An Introduction, Springer. $440 \mathrm{p}$.

$\mathrm{Ru}$ J, N Takeuchi, T Uezono, S Kaneta, M Minomura, T Takamura, A Higurashi, T Nakajima. 2000. Optical properties of biomass burning smoke in South-East Asia studied by NOAA/AVHRR and ground-base monitoring. Advances in Space Research 25(5): 1029-1032.

Sabins F. 1997. Remote Sensing: Principles and Interpretations. New York, U.S.A., WH Freeman. 432 p.

Setzer A, M Pereira. 1991. Operational detection of fires in Brazil with NOAA-AVHRR. Proc. 24th International Symposium on Remote Sensing of Environment. Río de Janeiro, Brazil, p. 469-482.

Siegert F, A Hoffmann. 2000. The 1998 Forest Fires in East Kalimantan (Indonesia): A Quantitative Evaluation Using High Resolution, Multitemporal ERS-2 SAR Images and NOAA-AVHRR Hotspot Data. Remote Sensing of Environment 72(1): 64-77.

Sifakis N, D Paronis, I Keramitsoglou. 2004. Combining AVHRR imagery with CORINE Land Cover data to observe forest fires and to assess their consequences. International Journal of Applied Earth Observation and Geoinformation 5(4): 263-274.

Sukhinin A, N French, E Kasischke, J Hewson, A Soja, I Csiszar, E Hyer, T Loboda, S Conrad, V Romasko, E Pavlichenko, S Miskiv, O Slinkina. 2004. AVHRR-based mapping of fires in Russia: New products for fire management and carbon cycle studies. Remote Sensing of Environment 93(4): 546-564.

Viedma O, E Chuvieco. 1993. Cartografía y evaluación de daños causados por incendios forestales mediante técnicas de teledetección. El ejemplo de la Hoya de Buñol (Valencia). AITIM Boletín de Información Técnica 167: 60-66. 
Wright R, L Flynn, H Gardeil, A Harris, E Pilger. 2002. Automated volcanic eruption detection using MODIS. Remote Sensing of Environment 82: 135-155.

Zhanqing L, Khananian, R Fraser, J Cihlar. 2001. Automatic detection of fire smoke using artificial neural networks and threshold approaches applied to AVHRR imagery.
IEEE Transactions on Geoscience and Remote Sensing 39(9): 1859-1870.

Zhukov B, E Lorenz, D Oertel, M Wooster, G Roberts. 2006. Spaceborne detection and characterization of fires during the bi-spectral infrared detection (BIRD) experimental small satellite mission (2001-2004). Remote Sensing of Environment 100(1): 51

Recibido: 04.05.06

Aceptado: 24.04.07 